\title{
ẢNH HƯởNG CỦA MẠNG XÃ HỘI FACEBOOK ĐẾN THÁI ĐỘ MUA HÀNG TRỤCC TƯYẾN CỦA GIỚI TRẺ TRONG THỜI KỲ CHUYỀN ĐỔI SỐ
}

\author{
BÙI THÀNH KHOA, HỒ NHẬT ANH, NGUYỄN MINH LÝ, NGUYỄN XUÂN TRƯỜNG \\ Khoa Thuong mai Du lịch, Truòng Đại học Công nghiệp thành phố Hồ Chí Minh \\ buithanhkhoa@iuh.edu.vn
}

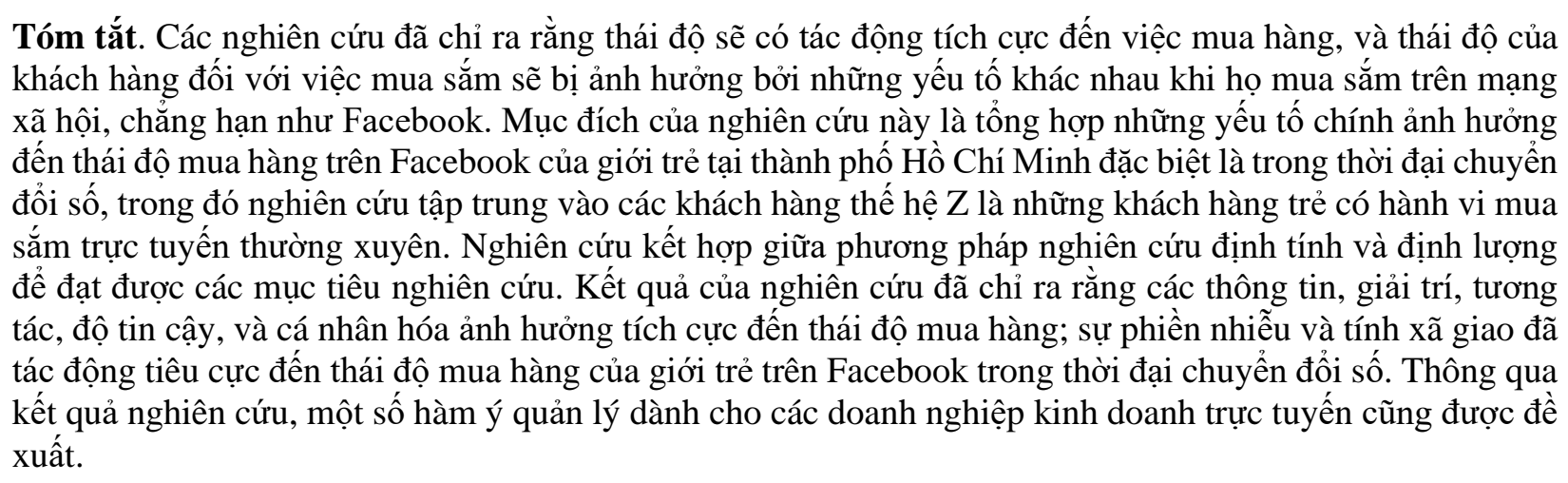

Từ khóa. thái độ mua hàng; mạng xã hội; tính xã giao; thông tin; giải trí; tương tác; độ tin cậy, cá nhân hóa; phiền nhiễu; chuyển đổi số

\section{THE IMPACT OF FACEBOOK ON YOUNG CUSTOMERS' BUYING ATTITUDE IN THE DIGITAL TRANSFORMATION AGE}

\begin{abstract}
Many studies has pointed out the attitude will have a huge impact on the customers' purchase, and the customer's attitude will be affected by other factors as shopping on social networks, i.e., Facebook. This research objective is to find out the key factors affecting the buying attitude of young customers on Facebook in the digital transformation age, especially this study focused on the Z Generation customer, who have frequent online buying behavior. This study combined qualitative and quantitative research methods to archive the research objectives. This study's result showed that informativeness, entertainment, interactivity, credibility, personalization had positive impact on the buying attitude; irritation and negative politeness had negative effect on the customers' buying attitudes in the digital transformation era. Based on the research result, some managerial implications for online businesses were also proposed.
\end{abstract}

Keywords: buying attitude, social network, negative politeness, informatinveness. entertaiment, interactivity, credibility, personalization, irritation, digital transformation.

\section{GIỚI THIỆ}

Facebook hiện có hơn 2,7 tỷ người dùng hoạt động hàng tháng tính đến quý 2 năm 2020 [1], là mạng xã hội lớn nhất trên toàn thế giới. Trong quý 3 năm 2012, số lượng người dùng Facebook tích cực đã vượt qua con số một tỷ, trở thành mạng xã hội đầu tiên từ trước đến nay. Facebook trở thành công cụ phổ biến để người dùng giao tiếp với bạn bè, đồng thời đây cũng là thị trường trực tuyến màu mỡ bên cạnh các nền tảng thương mại điện tử như website, hoặc ứng dụng di động [2]. Do tác động của chuyển đổi số, các cá nhân có thể chọn Facebook làm nơi bán hàng hoặc chọn Facebook để mua sắm nếu không thể mua sắm trực tiếp tại các cửa hàng. Theo các nghiên cứu trước đây, thái độ của khách hàng là yếu tố quan trọng nhất, sẽ ảnh hưởng đến hiệu quả kinh doanh của doanh nghiệp. Nếu khách hàng có thái độ tích cực thì việc thu hút khách hàng mua sắm trực tuyến trên Facebook sẽ dễ dành hơn, và lượng hàng hóa họ mua sắm sẽ tăng lên trong hiện tại và tương lai; do đó, các nhà bán hàng từ cá nhân đến doanh nghiệp hoạt động trên Facebook cần tập trung vào xây dựng thái độ tiêu dùng tích của người tiêu dùng [3, 4]. Hiện nay, các khách hàng trẻ tuổi đang có hành vi tiêu dùng trực tuyến mạnh mẽ. Tại Việt Nam, sự xuất hiện của làn sóng nhân khẩu học 
tiếp theo của người mua sắm ở Việt Nam - Thế hệ Z, thế hệ tiếp nối thế hệ Thiên niên kỷ (Millennials) - sẽ mang lại sự thay đổi lâu dài cho thị trường tiêu dùng trực tuyến [5].

Các nghiên cứu trước đây chủ yếu tập trung vào các phương tiện truyền thông như quảng cáo tin nhắn [6], hoặc quảng cáo trực tuyến [7], hoặc trang thương mại điện tử [8]. Các nghiên cứu về ảnh hưởng trực tiếp của các thuộc tính của Facebook đến thái độ của người dùng trên thị trường thương mại điện tử hiện tại vẫn còn hạn chế. Facebook là mạng xã hội với số lượng người sử dụng lớn, do đó quá trình cập nhật và thay đổi để tương thích với người dùng diễn ra liên tục. Các đặc điểm có lợi của Facebook được bổ sung liên tục để tạo ra tính hữu dụng cho người sử dụng như tính thông tin, tính giải trí, độ tin cậy [9-11], tính cá nhân hóa [9]. Bên cạnh đó, tương tác xã hội đã được nhiều nhà nghiên cứu xem là yếu tố dẫn đến hành vi mua hàng của khách hàng [12]. Xuất phát từ khái niệm chuẩn mực chủ quan trong lý thuyết hành vi hợp lý [13], thái độ đối với hành vi mua hàng của khách hàng dễ bị ảnh hưởng bởi các hành vi của cộng đồng mạng xã hội thông qua số lượng tương tác với bài viết hay sản phẩm; điều được thể hiện khi khách hàng nhấn nút "Thích", nút "Yêu thích", hoặc chia sẻ một bài đăng, hoặc bình luận về một bài đăng [14]. Tuy nhiên, những tác động này không phải lúc nào cũng tích cực, đáng kể khi bằng chứng có thể bị can thiệp bởi công nghệ hoặc mối quan hệ, hoặc do sự thiếu ý thức của người mua sắm khi tương tác với các bài viêt thông qua việc thích hay chia sẻ một cách tùy tiện dựa trên mối quan hệ hơn là các đặc điểm thật. Tính xã giao trong giao tiếp khi người giao tiếp chủ yếu thực hiện các hành vi buôn chuyện mà không chú ý đến nội dung [15]. Do đó, nghiên cứu này và kế thừa các nghiên cứu trước đó cũng bổ sung một yếu tố thích hợp cho môi trường Facebook, đó là tính xã giao thông qua giao tiếp ảnh hưởng đến thái độ của người mua hàng trên Facebook.

Bài báo này nhằm mục đích tìm ra mối quan hệ giữa tính thông tin, tính giải trí, tính tương tác, độ tin cậy, tính cá nhân hóa, sự phiền nhiễu, sự xã giao và thái độ mua hàng trên Facebook. Bên cạnh phần mở đầu, nghiên cứu này được tổ chức với phần tổng quan lý thuyết, phương pháp nghiên cứu, kết quả nghiên cứu, thảo luận và kết luận.

\section{TỔNG QUAN LÝ THUYẾT}

Thế hệ người tiêu dùng thế hệ Z, những người sinh từ năm 1998 đến 2010, sở hữu sức mạnh chi tiêu khổng lồ: họ ước tính chiếm $25 \%$ lực lượng lao động, và đạt tới 15 triệu người ở Việt Nam [16]. Thế hệ $\mathrm{Z}$ được lớn lên khi đất nước đang trải qua những thay đổi nhanh chóng, bao gồm cả sự bùng nổ kinh tế cùng với sự gia tăng của công nghệ và Internet [5]. Làn sóng nhân khẩu học đang nổi lên cũng được tiếp xúc với các tư tưởng phương Tây và quốc tế ở độ tuổi trẻ hơn so với các thế hệ trước. Do đó, việc mua sắm trực tuyến trở thành một thói quen thường của nhóm tuổi này, đồng thời việc tiếp cận với các mạng xã hội như Facebook, Zing, Instagram cũng trở thành một trong các hoạt động không thể thiếu hằng ngày của nhóm tuổi này. Các trang mạng xã hội nêu trên, đặc biệt là Facebook có ảnh hưởng mạnh đến thái độ cũng như hành vi tiêu dùng của thế hệ $Z$ [12]. Mạng xã hội trở thành một trong những công cụ hiệu quả nhất và phổ biến nhất trên thế giới hiện nay để tiếp cận với khách hàng. Bản chất của mạng xã hội là kết nối và chia sẻ. Tâm lý của người dùng mạng xã hội là muốn thể hiện bản thân, cái tôi của họ và mạng xã hội tạo ra một môi trường lý tưởng để thể hiện điều đó và hơn hết là thu hút sự chú ý. Bên cạnh đó, mạng xã hội cũng phát triển vì giải trí. Mọi người ngày càng tìm kiếm sự thú vị trong cuộc sống thông qua các hoạt động giải trí, và mạng xã hội là một trong những phương tiện giải trí tốt nhất hiện có để mang lại cho mọi người sự thoải mái và thư giãn. Lý thuyết hành vi hợp lý (TRA) giả định rằng hành vi con người được sự kiểm soát theo ý muốn, tức là mọi người tin rằng họ có thể thực hiện hành vi bất cứ khi nào họ sẵn sàng làm như vậy [13]. Dần dần, TRA được sử dụng thường xuyên hơn để nghiên cứu các hành vi mà sự kiểm soát là một yếu tố có thể thay đổi. Với mục đích đó, TRA đã được bổ sung bởi một thành phần được đặt tên là kiểm soát hành vi nhận thức. Khái niệm này thể hiện mức độ mà mọi người tin rằng họ có thể thực hiện hành vi vì họ có đủ năng lực. Một điều rất dễ nhận thấy là yếu tố này về cơ bản có thể cải thiện đáng kể tính tổng quát của việc áp dựng mô hình vì có nhiều hành vi cần kỹ năng cụ thể hoặc cơ sở vật chất bên ngoài. Kế thừa của TRA và bổ sung khái niệm kiểm soát hành vi là lý thuyết hành vi có kế hoạch (TPB). Tương tự như thái độ và chuẩn mực chủ quan, ngoài khả năng ảnh hướng đến ý định, nhận thức kiểm soát hành vi còn tác động đến hành vi cụ thể của một cá nhân [17]. 
Thái độ có thể được định nghĩa là cảm thấy thuận lợi hoặc không thuận lợi đối với một đối tượng, ảnh hưởng đến một người để hành động hoặc cư xử có thể đoán trước được đối với sản phẩm hoặc dịch vụ [18]. thái độ mua của người tiêu dùng được hiểu và mô tả xếp hạng tốt hoặc xấu dựa trên nhận thức, cảm xúc và khuynh hướng điều hành của một người về một đối tượng hoặc ý tưởng [19]. Sự phát triển của công nghệ đã tạo ra nhiều lợi thế cho mạng xã hội như: tính thông tin, tính giải trí, độ tin cậy, tính tương tác, tính cá nhân hóa và tính kích thích cho người dùng $[7,9]$. Các nghiên cứu trước đây chỉ ra rằng tính thông tin, tính giải trí, độ tin cậy, tính tương tác ảnh hưởng tích cực đển thái độ của người tiêu dùng và sự cáu kỉnh ảnh hưởng tiêu cực đến thái độ của người tiêu dùng. Hơn nữa, tính xã giao thông qua nhận xét hoặc đánh giá thường không ảnh hưởng đến thái độ của người tiêu dùng hoặc tác động tiêu cực [20]. Mô hình lý thuyết được thể hiện Hình 1.

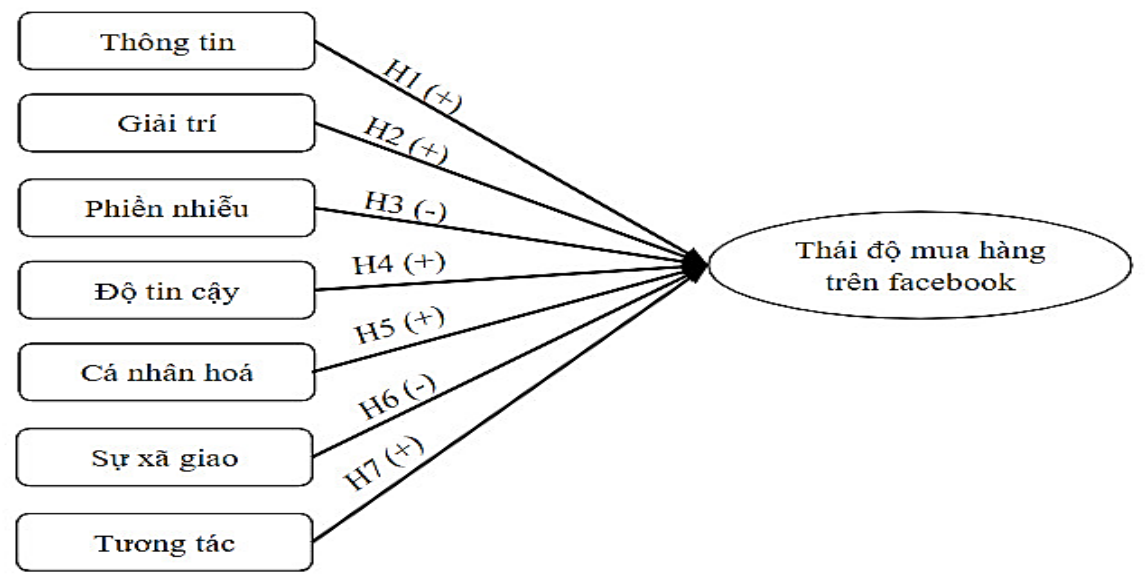

Hình 1. Mô hình nghiên cứu đề xuất

Tính thông tin cho thấy khả năng quảng cáo thông báo cho người tiêu dùng về sản phẩm và dịch vụ [21]. Tính thông tin là khả năng quảng cáo cung cấp thông tin liên quan một cách hiệu quả [22]. Người ta cũng nói rằng hiệu quả của việc cung cấp thông tin sẽ cho phép người tiêu dùng tìm kiếm thông tin mà không bị giới hạn về thời gian và địa điểm. Hiệu ứng này rất quan trọng đối với người tiêu dùng trên Internet [23]. Thông tin được coi là động lực có giá trị khiến người nhận phản ứng rất tích cực với các quảng cáo [7]. Do đó, nghiên cứu đề xuất giả thuyết:

H1: Tính thông tin có tác động tích cục đến thái độ mua hàng của khách hàng trẻ khi họ mua sắm trên Facebook.

Mức độ giải trí trên Facebook không chỉ quyết định đến sự thành công của quảng cáo được phân phối trên các phương tiện truyền thống, mà nó còn là yếu tố cần thiết để thành công trong bối cảnh Internet [24]. Khi xem xét các tài liệu có liên quan, người ta thấy rằng tính giải trí dùng để chỉ khả năng của quảng cáo đáp ứng nhu cầu của khán giả về thưởng thức thẩm mỹ, sự vui vẻ hoặc niềm vui cảm xúc. Nói cách khác, giải trí được coi là lợi ích của người tiêu dùng về mức độ thú vị, hài lòng khi họ thấy các quảng cáo [25]. Giải trí nhằm mục đích nâng cao sự hào hứng của người xem trên Facebook, thu hút nhiều lượt truy cập hơn và dẫn đến thái độ mua hàng của người tiêu dùng rất cao [26]. Do đó, nghiên cứu đề xuất giả thuyết:

H2: Tính giải trí có tác động tích cực đến thái độ mua hàng của khách hàng trẻ khi ho mua sắm trên Facebook.

Sự phiền nhiễu đề cập đến bất kỳ hiệu ứng tiêu cực nào có thể đi ngược lại những gì người dùng coi trọng [22]. Trong ngữ cảnh quảng cáo, sự phiền nhiễu được định nghĩa là việc gây khó chịu, xúc phạm, lăng mạ, lừa dối hoặc lôi kéo người xem quá mức [6]. Với mục đích tranh giành sự chú ý của người tiêu dùng, các chiến thuật xâm nhập của nhà quảng cáo có thể gây khó chịu cho khán giả [27]. Một quảng cáo quá phiền nhiễu sẽ dẫn đến thái độ tiêu cực cho khách hàng với thương hiệu đó. Một số khách hành cho rằng, học sẽ không thích mua sản phẩm bởi vì các quảng cáo quá nhiều của doanh nghiệp [22]. Do đó, nghiên cứu đề xuất giả thuyết:

H3: Sự phiền nhiễu có ảnh huởng tiêu cực đến thái độ mua hàng của khách hàng trẻ khi ho mua sắm trên Facebook. 
Độ tin cậy trong quảng cáo là một tuyên bố đáng tin và dễ nhận biết trong tâm trí người nghe [28]. Uy tín của quảng cáo bị ảnh hưởng bởi nhiều yếu tố khác nhau, đặc biệt là sự đáng tin của quảng cáo và danh tiếng của người đưa tin [3]. Độ tin cậy của quảng cáo được cảm nhận là một trong những cấu trúc đầu tiên ảnh hưởng đáng kể đến thái độ của người tiêu dùng đối với quảng cáo [6]. Do đó, giả thuyết $\mathrm{H} 4$ đã được đề xuất:

H4: Độ tin cậy có tác động tích cực đến thái độ mua hàng của khách hàng trẻ khi họ mua sắm trên Facebook Cá nhân hóa là quá trình điều chỉnh nội dung quảng cáo cho phù hợp với đặc điểm và sở thích của từng người tiêu dùng trên một trang thương mại điện tử cụ thể, có nghĩa là mỗi khách hàng được đối xử duy nhất khi mua sắm trên trang thương mại điện tử $[14,29]$. Cá nhân hóa nhằm mục đích cải thiện trải nghiệm của người dùng về một dịch vụ [30]. Do đó, tính cá nhân hóa có thể đảm bảo rằng khách truy cập vào các trang thương mại điện tử có thể tiếp xúc với các thông điệp quảng cáo phù hợp [31]; qua đó, họ sẽ bị hấp dẫn và nhận được những lợi ích tích cực từ việc quảng cáo trên trang thương mại điện tử. Do đó, sẽ là hợp lý nếu cho rằng cá nhân hóa của quảng cáo trên Facebook được coi là một yểu tố thiết yếu ảnh hưởng tích cực đến thái độ của người tiêu dùng đối với quảng cáo trên Facebook. Do đó, nghiên cứu đề xuất rằng:

H5: Cá nhân hóa có tác động tích cực đến thái độ mua hàng của khách hàng trẻ khi họ mua sắm trên Facebook

Theo lý thuyết của Brown và Levinson [15], lịch sự là trung tâm của hành vi tôn trọng. Tuy nhiên, sự khác biệt trong văn hóa Nhật Bản tập trung vào câuu trúc xã hội thứ bậc giữa người phát biểu và người nhận hơn là mong muốn của mọi người được tự do áp đặt như Brown và Levinson đề xuất [32]. Khi người tiếp nhận giả định có địa vị xã hội cao hơn hoặc thấp hơn, người phát biểu phải thừa nhận mối quan hệ xã hội này và thể hiện sự phụ thuộc của họ vào phát biểu, bằng cách đưa ra những áp đặt nghiêm khắc (nếu thấp hơn), hoặc bằng cách thể hiện hoặc sự xã giao đối với người nhận (nếu cấp trên). Ngày nay, hầu hết mọi người tương tác trong một thế giới ảo, nơi họ có thể nhìn thấy nhau qua màn hình máy tính hoặc giao tiếp bằng văn bản. Bối cảnh này đã thúc đẩy nhiều nghiên cứu chuyển trọng tâm sang giao tiếp máy tính. Facebook là một trong những mạng xã hội phổ biến nhất. Với 1,1 tỷ người dùng trên toàn thế giới, việc sử dụng Facebook làm phương tiện giao tiếp là điều không thể nghi ngờ. Do đó, hành vi xã giao có thể ảnh hưởng đến thái độ mua của người tiêu dùng, khả năng quay lại mua hàng sẽ thấp hơn trước nếu tính xã giao của các đánh giá quá cao [33]. Do đó, giả thuyết $\mathrm{H6}$ đã được đề xuất:

H6: Sư xã giao có ảnh hương tiêu cực đến thái độ mua hàng của khách hàng trẻ khi họ mua sắm trên Facebook

Tương tác là mức độ ảnh hưởng, và giao tiếp giữa người dùng với các loại quảng cáo khác nhau. Nghiên cứu trước đây đã chỉ ra sự tương tác trong ba nhóm: "người - người", "người - tin nhắn" và "người - máy tính" [9]. Đối với quảng cáo trực tuyến, mạng xã hội Facebook là một môi trường tiềm năng để quảng cáo bởi đây là môi trường mà sự tương tác giữa con người với con người ở mức cao nhất [34]. Do đó, thái độ mua hàng trực tuyến có thể bị ảnh hưởng bởi tính tương tác giữa những người sử dụng. Từ đó, nghiên cứu đề xuất giả thuyết $\mathrm{H} 7$ như sau:

H7: Tính tuoong tác có tác động tích cực đến thái độ mua hàng của khách hàng trẻ khi ho mua sắm trên Facebook.

\section{PHƯƠNG PHÁP NGHIÊN CÚU}

Phương pháp nghiên cứu hỗn hợp, bao gồm nghiên cứu định tính và nghiên cứu định lượng, được thực hiện để đạt được các mục tiêu nghiên cứu. Nghiên cứu định tính được thực hiện thông qua các buổi thảo luận nhóm tập trung. Nội dung thảo luận về các vấn đề đang nghiên cứu để thu thập ý kiến của các thành viên trong nhóm, bao gồm 10 thành viên có kinh nghiệm mua hàng trực tuyến trên Facebook. Buổi thảo luận nhóm tập trung được tiến hành dưới sự chủ trì của tác giả với dàn bài hướng dẫn thảo luận. Các biến quan sát được điều chỉnh sau nghiên cứu định tính sẽ mang lại giá trị khảo sát cao trong nghiên cứu định lượng. Sau khi hoàn thành thảo luận nhóm, bước tiếp theo là tiến hành nghiên cứu định lượng. Các mục quan sát được đo lường trên thang đo Likert 5 mức độ, bao gồm $1=$ rất không đồng ý; $2=$ không đồng ý; $3=$ trung tính; $4=$ đồng ý; 5 = rất đồng ý. Các thang đo được kế thừa từ các nghiên cứu trước đây; cụ thể, (1) tính thông tin bao gồm 4 mục được quan sát, (2) giải trí và (3) cá nhân hóa bao gồm 3 mục được quan sát, và (4) sự phiền nhiễu bao gồm 5 mục được ứng dụng từ Alsamydai [9]; (5) độ tin cậy và (6) tính tương tác bao gồm 4 mục được quan sát, lần lượt vận dụng thang đo của Aghakhani và cộng sự [10] và Abu-Ghosh và 
cộng sụ [11]; và (7) Tính xã giao bao gồm 4 mục được quan sát được đo lường bởi Hu và cộng sụx [35]; và cuối cùng là 5 mục được quan sát thái độ mua của Yaakop và cộng sụ [36]. Một cuộc khảo sát chính thức bằng cách chia sẻ bảng câu hỏi trực tuyến với những người sử dụng Facebook trên bất kỳ phương tiện truyền thông xã hội nào. Kết quả là có 325 đáp viên đều nói rằng họ đang sử dụng Facebook và trong đó có 290 người đã mua hàng trên Facebook. Như vậy, cỡ mẫu chính thức của nghiên cứu này là 290 mẫu; trong đó, $46,2 \%$ là nam và $53,8 \%$ là nữ. Đối tượng trả lời là thanh niên, độ tuổi từ 18 đến 22 tuổi, chiếm $92,6 \%$, đây cũng chính là nhóm tuổi chính của thế hệ $Z$. Đề tài phân tích dữ liệu thông qua việc sử dụng phần mềm SPSS để thực hiện các phân tích thống kề mô tả, kiểm định thang đo Cronbach's Alpha, phân tích nhân tố khám phá EFA, phân tích hồi quy đa biến.

\section{KẾT QUẢ VÀ THẢO LUẬN}

Giá trị trung bình của các biến quan sát trong Bảng 1 chỉ ra đánh giá của khách hàng về các yếu tố nghiên cứu. Kết quả trung bình chỉ ra rằng khách hàng coi quảng cáo bán hàng trên Facebook là phiền phức và không đáng tin cậy do ảnh hưởng trực tiếp từ bên ngoài.

Bảng 1. Thống kê mô tả

\begin{tabular}{|c|c|c|}
\hline Biến quan sát & $\begin{array}{c}\text { Kí } \\
\text { hiệu }\end{array}$ & $\begin{array}{l}\text { Trung } \\
\text { bình }\end{array}$ \\
\hline Facebook cung cấp nhiều thông tin về sản phẩm/dịch vụ. & INF1 & 3,74 \\
\hline Facebook cung cấp thông tin hữu ích về các thương hiệu, sản phẩm/dịch vụ. & INF2 & 3,66 \\
\hline Facebook liên tục cập nhật thông tin. & INF3 & 3,89 \\
\hline Tôi học được rất nhiều điều từ việc tiếp xúc với Facebook. & INF4 & 3,71 \\
\hline Tôi cảm thấy hứng thú với những nội dung, hình ảnh, video, bài viết,... trên Facebook. & ENT1 & 3,81 \\
\hline Những hình ảnh, video, bài viết,... trên Facebook thú vị. & ENT2 & 3,85 \\
\hline Những hình ảnh, video, bài viết,... về sản phẩm dịch vụ trên Facebook rất bắt mắt. & ENT3 & 3,74 \\
\hline Tôi cảm thấy phiền khi thấy những livestream bán hàng xuất hiện trên Facebook của tôi. & IRR1 & 2,17 \\
\hline $\begin{array}{l}\text { Tôi cảm thấy mất thời gian khi một quảng cáo chen ngang lúc tôi đang xem video trên } \\
\text { Facebook. }\end{array}$ & IRR2 & 1,98 \\
\hline Tôi cảm thấy khó chịu khi nhìn thấy quảng cáo trên Facebook. & IRR3 & 2,24 \\
\hline Quảng cáo qua mạng xã hội Facebook làm tôi phân tâm khỏi nội dung khác. & IRR4 & 2,37 \\
\hline $\begin{array}{l}\text { Tôi cảm thấy KHÔNG hài lòng khi các nội dung bạo lực, phản cảm xuất hiện trên Facebook } \\
\text { của tôi. }\end{array}$ & IRR5 & 2,06 \\
\hline Tôi sử dụng Facebook làm nơi tham khảo để mua sản phẩm hoặc dịch vụ. & CRE1 & 3,37 \\
\hline $\begin{array}{l}\text { Nội dung về sản phẩm/dịch vụ trên Facebook khá thiết thực, một phần xuất phát từ những } \\
\text { người đã biết. }\end{array}$ & CRE2 & 3,51 \\
\hline Sản phẩm/dịch vụ trên Facebook đáng tin cậy. & CRE3 & 3,04 \\
\hline Nội dung về sản phẩm/dịch vụ trên Facebook thuyết phục. & CRE4 & 3,25 \\
\hline Đề xuất trên Facebook phù hợp với sở thích của tôi. & PER1 & 3,63 \\
\hline Nội dung trên Facebook gần với chủ đề mà tôi quan tâm. & PER2 & 3,72 \\
\hline Nội dung của sản phẩm/dịch vụ trên Facebook đúng như những gì tôi mong đợi. & PER3 & 3,56 \\
\hline Những sản phẩm/dịch vụ được đánh giá tốt trên Facebook thì KHÔNG thật sự chất lượng. & IMP1 & 2,75 \\
\hline Nội dung, lượt thích, chia sẻ, bình luận của các bài viết trên Facebook thì KHÔNG thực tế. & IMP2 & 2,7 \\
\hline Những bình luận về sản phẩm/dịch vụ trên Facebook KHÔNG hoàn toàn tự nhiên. & IMP3 & 2,52 \\
\hline $\begin{array}{l}\text { Các bình luận/đánh giá trên Facebook bị ẢNH HƯỞNG bởi các yếu tố khách quan (theo số } \\
\text { đông, theo xu hướng). }\end{array}$ & IMP4 & 2,31 \\
\hline Facebook mang lại mức độ nhận biết về sản phẩm/dịch vụ cao. & INT1 & 3,57 \\
\hline
\end{tabular}




\begin{tabular}{|l|l|c|}
\hline \multicolumn{1}{|c|}{ Biến quan sát } & $\begin{array}{c}\text { Kí } \\
\text { hiệu }\end{array}$ & $\begin{array}{c}\text { Trung } \\
\text { bình }\end{array}$ \\
\hline Facebook giúp tôi tiếp cận dễ dàng hơn sản phẩm/dịch vụ. & INT2 & 3,75 \\
\hline Tôi thường nhấp vào nội dung bài viết, quảng cáo để hiểu rõ hơn sản phẩm/dịch vụ. & INT3 & 3,59 \\
\hline Tôi trao đổi với người bán thông qua tin nhắn riêng hoặc bình luận trên bài viết. & INT4 & 3,75 \\
\hline Tôi thích đọc các nội dung về sản phẩm/dịch trên Facebook. & ATT1 & 3,36 \\
\hline Tôi muốn xem các sản phẩm/dịch vụ trên Facebook (trước khi đến cửa hàng). & ATT2 & 3,47 \\
\hline Tôi thích thú với những giá trị cộng đồng mà Facebook mang lại. & ATT3 & 2,96 \\
\hline Tôi xem Facebook là lựa chọn tốt để mua hàng online. & ATT4 & 3,53 \\
\hline $\begin{array}{l}\text { Tôi phản ứng tích cực với những nội dung về sản phẩm/dịch vụ trên Facebook đã gợi ý cho } \\
\text { tôi. }\end{array}$ & ATT5 & 3,26 \\
\hline
\end{tabular}

Nghiên cứu này đã kiểm tra độ tin cậy và giá trị hội tụ thông qua Cronbach's Alpha và phân tích nhân tố khám phá (EFA) sử dụng phương pháp phân tích thành phần chính với phép quay Varimax để giảm các biến quan sát thành một tập hợp các cấu trúc khác nhau. Tất cả giá trị Cronbach's Alpha phải lớn hơn 0,7 để đảm bảo độ tin cậy của thang đo. Bảng 2 cho thấy thang đo của tất cả các công trình có ý nghĩa lớn hơn 0,7 ; do đó, tất cả các thang đo đều đáng tin cậy. Hơn nữa, hệ số $\mathrm{KMO}=0,908$, hơn 0,$5 ;$ do đó, EFA là thích hợp. Trong kiểm định Bartlett, Sig. $=0,00$, nhỏ hơn 0,05 , chỉ ra rằng tất cả các biến quan sát đều có tương quan. Hơn nữa, hệ số tải của tất cả các mục có ý nghĩa và giá trị lớn hơn 0,5 . Tất cả các thang đo trong nghiên cứu này đều có giá trị hội tụ.

Bảng 2. Kết quả phân tích Cronbach’s Alpha và hệ số khám phá (EFA)

\begin{tabular}{|c|c|c|c|}
\hline Cấu trúc nghiên cứu & Cronbach's Alpha & Hệ số tải & Một số chỉ số kiểm định EFA \\
\hline Tính cá nhân hoá (PER) & 0,913 & {$[0,833-0,911]$} & \multirow{7}{*}{$\begin{array}{ll}\text { - } & \text { KMO }=0,908 \\
\text { - } & \text { Sig. }(\text { Bartlett's Test of } \\
& \text { Sphericity) }=0,00 \\
\text { - } & \text { Eigenvalue }=1,115 ; \\
\text { - } & \text { Tổng phương sai trích = } \\
75,194\end{array}$} \\
\hline Tính thông tin (INF) & 0,878 & {$[0,828-0.865]$} & \\
\hline Sự phiền nhiễu (IRR) & 0,884 & {$[0,841-0,870]$} & \\
\hline Sự xã giao (IMP) & 0,864 & {$[0,806-0,853]$} & \\
\hline Tính tương tác (INT) & 0,876 & {$[0,820-0,865]$} & \\
\hline Độ tin cậy (CRE) & 0,872 & {$[0,823-0,849]$} & \\
\hline Tính giải trí (ENT) & 0,874 & {$[0,791-0,849]$} & \\
\hline
\end{tabular}

Kết quả phân tích hồi quy tuyến ở Bảng 3 cho thấy kiểm định giả thuyết "Tất cả các hệ số của mô hình đều bằng không", kết quả phân tích phương sai cho thấy Sig. $=0,000<0,05$ điều đó có nghĩa là có ít nhất một hệ số hồi quy khác không nên bác bỏ giả thuyết trên và mô hình phù hợp. Từ bảng 3 cho thấy hệ số phóng đại phương sai $(\mathrm{VIF})$ nhỏ hơn 2 (lớn nhất là $\mathrm{VIF}=1,998<2$ ), do đó, hiện tượng đa cộng tuyến giữa các biến độc lập trong mô hình này là nhỏ, không có ảnh hưởng đáng kể đến kết quả hồi quy. Tham số $\mathrm{R}^{2}$ điều chỉnh cho biết mức độ $(\%)$ của biến phụ thuộc được giải thích bởi biến độc lập. Theo bảng $3, \mathrm{R}^{2}$ điều chỉnh $=85,8 \%$, có nghĩa là $85,8 \%$ sự biến đổi của biến phụ thuộc ATT được giải thích bởi sự biến đổi của 7 yếu tố độc lập bao gồm INF, ENT, IRR, CRE, PER, IMP, INT; các yếu tố khác ngoài mô hình giải thích 14,2\% sự thay đổi còn lại. Hệ số Durbin - Watson $(\mathrm{d})=1,933$, với kích thước mẫu $\mathrm{N}=290$, ta có $\mathrm{dl}=1,344<$ $1,933<(4-1,246)=2,754$, do đó thỏa mãn điều kiện nên mô hình không có sự tự tương quan phần dư. Bảng phân tích ANOVA một chiều cho thấy $\mathrm{F}=250,444$ và mức ý nghĩa Sig. $=0,000($ sig. $\leqslant 0,05)$, nghĩa là mô hình hồi quy phù hợp với dữ liệu thu thập và các biến được đưa vào có ý nghĩa thống kê với mức ý nghĩa 5\%.

Bảng 4. Kết quả hồi quy

\begin{tabular}{|c|c|c|c|c|c|c|c|}
\hline & Hệ số B & Sai số chuẩn & Beta & sig. & VIF & Giả thuyết & Kết luận \\
\hline Hằng số & 2,042 &, 138 & & 0,000 & & & \\
\hline INF &, 119 &, 024 &, 154 & 0,000 & 1,998 & H1 & Chấp nhận \\
\hline ENT &, 057 &, 022 &, 075 & 0,000 & 1,669 & H2 & Chấp nhận \\
\hline
\end{tabular}



CỦA GIỚI TRẺ TRONG THỜI KỲ CHUYỂN ĐỔI SỐ

\begin{tabular}{|c|c|c|c|c|c|c|c|}
\hline IRR &,- 138 & 018 &,- 196 & 0,000 & 1,395 & H3 & Chấp nhận \\
\hline CRE &, 185 & 021 & ,263 & 0,000 & 1,806 & $\mathrm{H} 4$ & Chấp nhận \\
\hline PER & 068 & 019 & 095 & 0,000 & 1,461 & $\mathrm{H} 5$ & Chấp nhận \\
\hline IMP &,- 208 &, 020 &,- 287 & 0,000 & 1,598 & H6 & Chấp nhận \\
\hline INT & ,159 & 024 & 206 & 0,000 & 1,945 & $\mathrm{H} 7$ & Chấp nhận \\
\hline \multicolumn{2}{|c|}{$\mathrm{R}^{2}$ điều chỉnh $=0,858$} & \multicolumn{2}{|c|}{ Durbin-Watson $=1,933$} & \multicolumn{2}{|c|}{$F=250,444$} & \multicolumn{2}{|c|}{ sig. $($ ANOVA $)=0,00$} \\
\hline
\end{tabular}

Hệ số Beta ở bảng 4 cho thấy mức độ ảnh hưởng của 7 biến độc lập đến biến phụ thuộc và tầm quan trọng của từng biến độc lập trong mô hình như sau: $\mathrm{INF}=0,154, \mathrm{ENT}=0,075, \mathrm{IRR}=-0,196, \mathrm{CRE}=0,263$, PER $=0,095, \mathrm{IMP}=-0,287, \mathrm{INT}=0,206$. Kết quả nghiên cứu cho thấy rằng tất cả các giá trị có ý nghĩa đều nhỏ hơn 0,05 , vì vậy tất cả các biến độc lập tác động đáng kể đến thái độ mua của giới trẻ với độ tin cậy $99 \%$. Với kết quả ở bảng 4,phương trình hồi quy được thiết lập như sau:

ATT $=-0,138 * I R R+0,185 *$ CRE + 0,068*PER - 0,208*IMP + 0,057*ENT + 0,119*INF + 2,042.

\section{THẢO LUẬN VÀ HÀM Ý QUẢN TRI}

\subsection{Thảo luận}

Nghiên cứu này nhằm xác định các yếu tố của Facebook ảnh hưởng đến thái độ mua hàng của người tiêu dùng, đặc biệt là giới trẻ. Kết quả nghiên cứu định tính và khảo sát định lượng với quy mô mẫu gồm 290 người tham gia nhằm kiểm tra mô hình hồi quy tuyến tính, trong đó, thái độ mua hàng bị ảnh hưởng bởi các yếu tố như: Tính thông tin, Sự phiền nhiễu, Sự tin cậy, Tính cá nhân hóa, Tính xã giao, tính tương tác, và tính giải trí. Một số đóng góp về lý luận và thực tiễn được nêu ra như sau:

Một là tính thông tin của Facebook luôn có tác động tích cực và có ảnh hưởng đáng kể đến đến thái độ mua hàng $($ Beta $=0,154$, sig. $=0,000)$. Do đó, việc cung cấp thông tin thường xuyên là lợi thế chính của việc thu hút khách hàng tiềm năng và nuôi dưỡng cơ sở khách hàng của doanh nghiệp [21,37]. Sự tin tưởng của người tiêu dùng có thể có được bằng kinh nghiệm bản thân hoặc do người quen giới thiệu [38]. Vì vậy, các doanh nghiệp bán lẻ trực tuyến cần thường xuyên giao tiếp, tạo sự gần gũi và tin tưởng đối với sản phẩm và thương hiệu của mình. Thứ hai, sự phiển nhiễu đã tác động tiêu cực đến thái độ mua sắm, với Beta = 0,196 , sig. $=0,000$. Nhiều quảng cáo về sản phẩm cũng không nhất định là tốt, điều đó gây ra sự phiền nhiễu cho khách hàng và tác động tiêu cực đến thái độ của họ $[6,39]$. Vì vậy, khi tham gia kinh doanh trên Facebook, doanh nghiệp cần lưu ý đến tính chất của nội dung; nội dung cần đúng trường hợp và tăng hiệu quả. Do đó, người tiêu dùng càng cảm thấy khó chịu, bức xúc trước nội dung mà quảng cáo trên mạng xã hội mang lại thì họ sẽ không còn ý định mua sản phẩm được quảng cáo, làm giảm hiệu quả quảng cáo trên Facebook.Thứ ba, sự tin cậy cũng là một trong những yếu tố tác động tích cực đến thái độ mua hàng (Beta $=0,263$, sig. $=0,000$ ). Trong môi trường mà sản phẩm không thể chạm trước, hoặc quá nhiều các bình luận không chính xác, sự tin cậy trở thành yếu tố quan trọng tác động đến thái độ của người tiêu dùng $[6,39]$. Qua đó, doanh nghiệp cần tạo dựng được niềm tin với khách hàng, uy tín sẽ mang lại lợi ích lâu dài cho doanh nghiệp. Quảng cáo và bán hàng trên Facebook là trung thực vì nó một phần đến từ những người đã biết về thông tin sản phẩm/dịch vụ/thương hiệu; do đó, họ sẽ dễ dàng tin tưởng.

Thứ tư, cá nhân hóa có tác động tích cực đến thái độ mua sắm trên Facebook (Beta = 0,095, sig. = 0,000). cá nhân hóa sẽ tạo ra sự lựa chọn hoặc chương trình cụ thể và khác biệt cho từng tập khách hàng, giúp giảm chi phí nhưng mang lại hiệu quả cao [30]. Việc hiểu rõ từng phân khúc khách hàng là rất quan trọng vì khi có sự tập hợp của từng nhóm đối tượng, doanh nghiệp có thể đưa ra lời kêu gọi hành động chính xác và phù hợp hơn [40]. Nếu doanh nghiệp nắm bắt thông tin của từng phân khúc đối tượng thông qua các hành vi trực tuyến, sẽ có thể cung cấp những đề xuất phù hợp cho người tiêu dùng trong mua hàng trực tuyến, qua đó sẽ giúp doanh nghiệp tinh chỉnh chiến lược và tối ưu hóa đối tượng mục tiêu. Đồng thời, doanh nghiệp có thể tạo ra những trải nghiệm độc đáo, khác biệt giữa một cá nhân và một thương hiệu cụ thể.

Thứ năm, sự xã giao ảnh hưởng tiêu cực đến thái độ mua hàng trên Facebook (Beta = - 0,287, sig. = 0,000). Mặc dù một bức ảnh hoặc trạng thái trên Facebook được đăng trên trang của một người có tính tương tác cao, sự lan truyền có thể đến với hàng triệu người dùng Facebook khác; tuy nhiên đa phần khách hàng cũng không có sự tin tưởng vào những đăng tải này [15]. Hiện tại, các nội dung đăng tải trên Facebook của người dùng hiện nay không còn chỉ gói gọn trong những câu chuyện đời tư mà đôi khi họ còn chia sẻ nhiều thứ 
khác, có thể là một bài báo, một câu chuyện yêu thích. Trong những năm gần đây, khi nội dung trên Facebook đã ảnh hưởng không nhỏ đến đời sống thực tế xã hội, gây hoang mang dư luận hoặc khiến người tiêu dùng hiểu nhầm và có những hành vi mua hàng không đúng với nhu cầu [35]. Các bình luận cẩu thả, qua loa mang tính chất xã giao hoặc để nhận quà từ doanh nghiệp đang khiến người tiêu dùng mất lòng tin vào các đánh giá trực tuyến, và xem đó như là xã giao, chiếu lệ, và thiếu tính khách quan.

Thứ sáu, tính tương tác như chia sẻ, bình luận, hoặc trao đổi thông tin qua mạng xã hội có ảnh hưởng tích cực đến thái độ của người tiêu dùng trên Facebook (Beta $=0,206$, sig. $=0,000)$. Kết quả nghiên cứu chứng minh rằng người tiêu dùng sẽ có thái độ mua hàng cao hơn nếu họ xem quảng cáo thông qua mạng xã hội [9]. Tương tác xã hội được thể hiện qua việc người dùng có thể sử dụng nhiều văn bản, hình ảnh, video và liên kết khác nhau để theo dõi và chia sẻ sản phẩm mới với những người dùng khác [34]. Đồng thời, các thông điệp mang tính tương tác cao đến cộng đồng xã hội và các cá nhân có ảnh hưởng sẽ tác động đáng kể đển thái độ của người tiêu dùng thông qua các bình luận, và chia sẻ nội dung quảng cáo . Các doanh nghiệp cần tận dụng lợi thế này để tăng tỷ lệ chuyển đổi và lòng trung thành của khách hàng khi truyền thông qua mạng xã hội, đặc biệt là Facebook [4].

Cuối cùng, tính giải trí tác động tích cực đến thái độ mua hàng (Beta $=0,075$, sig. $=0,000$ ). Facebook mang lại nhiều giá trị thích thú cho người dùng và khách hàng. Các doanh nghiệp có thể tận dụng điều này để có chiến lược tiếp cận khôn ngoan hơn. Theo đó, nếu một quảng cáo thông qua mạng xã hội mang tính giải trí đến người tiêu dùng nhiều nhất có thể, thì thái độ của của họ sẽ tăng lên. Có thể thấy, yêu cầu cơ bản và bắt buộc đối với các chương trình và hoạt động quảng cáo là khả năng cung cấp thông tin và giải trí cho người xem [6]. Chỉ khi người tiêu dùng cảm nhận được hai giá trị này, họ mới có thể tạo ra một thái độ tích cực đối với quảng cáo. tính giải trí của Facebook được đo lường bằng những hoạt động mang lại niềm vui, sự thoải mái cho người tiêu dùng và thể hiện ở nội dung mà quảng cáo truyền tải [29]. Trang bán hàng cần xây dựng tinh tế, để thể hiện sự đơn giản, dễ hiểu và độc đáo; đây cũng là cách giúp người dùng không cảm thấy nhàm chán khi tiếp nhận thông tin quảng cáo từ các trang mạng xã hội, qua đó sẽ tác động tích cực đến thái độ mua hàng của các khách hàng trẻ thuộc thế hệ $\mathrm{Z}$.

\subsection{Hàm ý quản trị}

Các trang thương mại điện tử tốt nhất trên thế giới đã thực hiện tích hợp với mạng xã hội. Các kênh truyền thông xã hội như một công cụ làm cơ sở để tăng tính tương tác - giống như các đại diện bán hàng khu vực trong môi trường cửa hàng ảo. Mua sắm trên Facebook đang trở thành một xu hướng mới trong thương mại trực tuyến. Khách hàng cảm thấy thuận tiện với tất cả quá trình mua sắm được thực hiện trong một ứng dụng. Họ quan tâm đến sản phẩm thông qua chia sẻ từ bạn bè hoặc quảng cáo trên Facebook, sau đó tiếp tục quá trình mua sắm mà không cần chuyển đổi ứng dụng, điều có thể mất thời gian khi đăng nhập, họ thường gặp sự cố và trải qua quá trình đặt lại mật khẩu. Sự linh hoạt của dịch vụ hô̂ trợ khách hàng là một lý do khác khiến khách hàng thích mua sắm trên Facebook. Người tiêu dùng rất quan tâm đến quy trình và việc tạo ra các yêu cầu kiểm tra tình trạng đơn hàng, thay đồi thời gian hoặc địa điểm giao hàng. Lợi thế này giúp họ dễ dàng mua sắm trên Facebook vì bộ phận hố trợ khách hàng của cửa hàng luôn sẵn sàng phản hồi ngay lập tức để giải quyết yêu cầu của họ. Nghiên cứu đã chỉ ra mối quan hệ tiêu cực giữa sự phiền nhiễu, sự xã giao; và mối quan hệ tích cực giữa thông tin, độ tin cậy, cá nhân hóa, tính tương tác và tính giải trí.

Nghiên cứu được thực hiện một cách khoa học và đầy đủ nhất có thể; tuy nhiên, nó vẫn có một số hạn chế. Thứ nhất, nghiên cứu mới chỉ thực hiện những thử nghiệm chung về khảo sát thực tế của giới trẻ trên mạng xã hội Facebook, mặc dù Facebook là một trang mạng xã hội tương đối đầy đủ với nhiều loại thông tin từ giáo dục, $\mathrm{y}$ tế. Thứ hai, về phương pháp chọn mẫu, do thời gian và khung dữ liệu, tác giả chỉ có thể chọn mẫu ngẫu nhiên, điều này làm giảm độ tin cậy của nghiên cứu. Thứ ba, nghiên cứu chỉ tập trung vào ảnh hưởng của Facebook đối với thái độ mua hàng của giới trẻ trong quá trình chuyển đổi kỹ thuật số. Các nghiên cứu tiếp theo có thể khắc phục một số hạn chế của nghiên cứu này bằng cách tập trung vào một yếu tổ ảnh hưởng rõ ràng đến tính cụ thể của mạng xã hội đó hoặc các nền tảng truyền thông xã hội khác. Bên cạnh đó, có thể xây dựng khung lấy mẫu điều tra theo phương pháp xác suất để tạo độ tin cậy nghiên cứu cao hơn. Một số biến nghiên cứu có thể được nâng cao để tạo ra nghiên cứu sâu rộng, đặc biệt là các biến nghiên cứu về các yếu tố ảnh hưởng đến quyết định mua hàng trên mạng xã hội, tác động đến ý định mua, mua lặp lại hoặc yếu tố ảnh hưởng đến lòng trung thành của khách hàng thông qua quảng cáo lạ̣p lại trên mạng xã hội. 


\section{TÀI LIỆU THAM KHẢO}

[1] J. Clement. (2020, November 29). Facebook: number of monthly active users worldwide 2008-2020. Truy cập tại: https://www.statista.com/statistics/264810/

[2] R. Mosquera, M. Odunowo, T. McNamara, X. Guo, và R. Petrie, The economic effects of Facebook, Experimental Economics, vol. 23, no. 2, pp. 575-602, 2020.

[3] A. J. Fredricks và D. L. Dossett, Attitude-behavior relations: A comparison of the Fishbein-Ajzen and the Bentler-Speckart models, Journal of personality and social psychology, vol. 45, no. 3, p. 501, 1983.

[4] B. T. Khoa, Ảnh hưởng của mạng xã hội đến lòng trung thành của khách hàng: Úng dụng trường hợp của Facebook, Tạp chí Khoa học - Công nghệ, vol. 18, no. 1, pp. 86-97, 2015.

[5] B. T. Khoa, "The role of Mobile Skillfulness and User Innovation toward Electronic Wallet Acceptance in the Digital Transformation Era," in 2020 International Conference on Information Technology Systems and Innovation (ICITSI), Bandung - Padang, Indonesia, 2020, pp. 30-37: IEEE, 2020.

[6] N. M. N. Najiba, J. Kasumab, và Z. B. H. Bibic, "Relationship and Effect of Entertainment, Informativeness, Credibility, Personalization and Irritation of Generation Y's Attitudes towards SMS Advertising," presented at the Proceedings of the 3rd International Conference on Business and Economics, Shah Alam, Malaysia, 21-23 September 2016, 2016.

[7] S. K. Ariffin, T. L. Aun, và Y. Salamzadeh, How Personal Beliefs Influence Consumer Attitude towards Online Advertising in Malaysia: To Trust or Not to Trust?, Global Business \& Management Research, vol. 10, no. 1, 2018.

[8] Y. Gao và X. Wu, A cognitive model of trust in e-commerce: Evidence from a field study in China, Journal of Applied Business Research (JABR), vol. 26, no. 1, 2010.

[9] M. J. Alsamydai, Adaptation of the technology acceptance model (TAM) to the use of mobile banking services, international review of management and business research, vol. 3, no. 4, p. 2039, 2014.

[10] N. Aghakhani, J. Karimi, và M. Salehan, A unified model for the adoption of electronic word of mouth on social network sites: Facebook as the exemplar, International Journal of Electronic Commerce, vol. 22, no. 2, pp. 202-231, 2018.

[11] D. H. Abu-Ghosh, H. Al-Dmour, A. A. Alalwan, và R. H. Al-Dmour, "Factors affecting Jordanian consumers' attitudes towards Facebook advertising: case study of tourism," in Emerging Markets from a Multidisciplinary Perspective: Springer, 2018, pp. 285-302.

[12] B. T. Khoa và H. M. Nguyen, Electronic Loyalty In Social Commerce: Scale Development and Validation, Gadjah Mada International Journal of Business, vol. 22, no. 3, 2020. doi: 10.22146/gamaijb.50683

[13] I. Ajzen và M. Fishbein, Belief, attitude, intention and behavior: An introduction to theory and research. Reading, MA: Addison-Wesley, 1975.

[14] M. H. Nguyen và B. T. Khoa, Perceived Mental Benefit in Electronic Commerce: Development and Validation, Sustainability, vol. 11, no. 23, pp. 6587-6608, 2019. doi: 10.3390/su11236587

[15] P. Brown và S. C. Levinson, Politeness: Some universals in language usage. Cambridge: Cambridge university press, 1987. 
[16] M. Kyssha. (2019, 15/10). Vietnam's New Age Consumers: Generation Z. Truy cập tại: https://www.vietnambriefing.com/news/vietnams-new-age-consumers-generation-z.html

[17] I. Ajzen, From intentions to actions: A theory of planned behavior, Kuhl J., \& Beckmann J. ed. (Action Control. SSSP Springer Series in Social Psychology). Berlin, Germany: Springer, 1985, pp. 11-39.

[18] B. T. Khoa, The Impact of the Personal Data Disclosure's Tradeoff on the Trust and Attitude Loyalty in Mobile Banking Services, Journal of Promotion Management, vol. 27, no. 4, pp. 585-608, 2020. doi: $10.1080 / 10496491.2020 .1838028$

[19] B. Knezevic, M. Falat, và I. S. Mestrovic, Differences Between X and Y Generation in Attitudes Towards Online Book Purchasing, Journal of Logistics, Informatics and Service Science, vol. 7, no. 1, pp. 1-16, 2020. doi: 10.33168/liss.2020.0101

[20] P. Brown, "Politeness and impoliteness," in The Oxford handbook of pragmatics, H. Yan, Ed., Oxford, United Kingdom: Oxford University Press, 2019, pp. 383-399.

[21] M. Al Khasawneh và A. Shuhaiber, A comprehensive model of factors influencing consumer attitude towards and acceptance of SMS advertising: an empirical investigation in Jordan, International Journal of Sales \& Marketing Management Research and Development, vol. 3, no. 2, pp. 1-22, 2013.

[22] L.-B. Oh và H. Xu, Effects of multimedia on mobile consumer behavior: An empirical study of locationaware advertising, ICiS 2003 Proceedings, p. 56, 2003.

[23] L.-d. Chen và R. Nath, A framework for mobile business applications, International Journal of Mobile Communications, vol. 2, no. 4, pp. 368-381, 2004.

[24] R. H. Ducoffe, How consumers assess the value of advertising, Journal of Current Issues \& Research in Advertising, vol. 17, no. 1, pp. 1-18, 1995.

[25] A. A. Raney và J. Bryant, "Entertainment and Enjoyment as Media Effect," in Media Effects: Advances in Theory and Research, M. B. Oliver, A. A. Raney, and J. Bryant, Eds., New York: N.Y. Routledge, Taylor \& Francis Group 2019.

[26] D. R. Van der Waldt, T. Rebello, và W. Brown, Attitudes of young consumers towards SMS advertising, African Journal of Business Management, vol. 3, no. 9, pp. 444-452, 2009.

[27] M. Y. Cheung, C. Luo, C. L. Sia, và H. Chen, Credibility of Electronic Word-of-Mouth: Informational and Normative Determinants of On-line Consumer Recommendations, International Journal of Electronic Commerce, vol. 13, no. 4, pp. 9-38, 2014. doi: 10.2753/jec1086-4415130402

[28] Y.-H. Fang, Beyond the Credibility of Electronic Word of Mouth: Exploring eWOM Adoption on Social Networking Sites from Affective and Curiosity Perspectives, International Journal of Electronic Commerce, vol. 18, no. 3, pp. 67-102, 2014. doi: 10.2753/jec1086-4415180303

[29] M. H. Nguyen và B. T. Khoa, Customer Electronic Loyalty towards Online Business: The role of Online Trust, Perceived Mental Benefits and Hedonic Value, Journal of Distribution Science, vol. 17, no. 12, pp. 81-93, 2019. doi: 10.15722/jds.17.12.201912.81

[30] D. Tynan. (2018, June 12). Personalization Is a Priority for Retailers, but Can Online Vendors Deliver? Truy cập tại: https://www.adweek.com/digital/personalization-is-a-priority-for-retailers-online-and-off-but-itsharder-than-it-looks-in-an-off-the-shelf-world/ 
[31] P.-T. Chen và H.-H. Hu, The effect of relational benefits on perceived value in relation to customer loyalty: An empirical study in the Australian coffee outlets industry, International journal of hospitality management, vol. 29, no. 3, pp. 405-412, 2010.

[32] Y. Matsumoto, Reexamination of the universality of face: Politeness phenomena in Japanese, Journal of pragmatics, vol. 12, no. 4, pp. 403-426, 1988.

[33] N. Sukpanich và L.-d. Chen, Interactivity as the driving force behind e-commerce, AMCIS 2000 Proceedings, p. 244, 2000.

[34] X. Wang, C. Yu, và Y. Wei, Social media peer communication and impacts on purchase intentions: A consumer socialization framework, Journal of interactive marketing, vol. 26, no. 4, pp. 198-208, 2012.

[35] Y. Hu, A. Tafti, và D. Gal, "Read This, Please? The Role of Politeness in Customer Service Engagement on Social Media," presented at the Proceedings of the 52nd Hawaii International Conference on System Sciences, Grand Wailea, Hawaii, 2019.

[36] A. Yaakop, M. Mohamed Anuar, K. Omar, và A. Liaw, "Consumers' perceptions and attitudes towards advertising on Facebook in Malaysia," in World Business and Economics Research Conference, Auckland New Zealand, 2012.

[37] R. K. Gharib, A. Garcia-Perez, S. Dibb, và Z. Iskoujina, Trust and reciprocity effect on electronic word-ofmouth in online review communities, Journal of Enterprise Information Management, vol. 33, no. 1, pp. 120-138, 2019. doi: 10.1108/jeim-03-2019-0079

[38] P. Kotler, G. Armstrong, và M. O. Opresnik, Principles of Marketing. Harlow, England: Pearson, 2021.

[39] R. Cheung, The influence of electronic word-of-mouth on information adoption in online customer communities, Global Economic Review, vol. 43, no. 1, pp. 42-57, 2014.

[40] D. Riecken, Personalized views of personalization, Communications of the ACM, vol. 43, no. 8, pp. 26-26, 2000. doi: https://doi.org/10.1145/345124.345133

Ngày nhận bài: 29/04/2021

Ngày chấp nhận đăng: 23/06/2021 